\title{
Development of a Culturally Sensitive Asian American/Pacific Islander Curriculum for Child Psychiatry Trainees
}

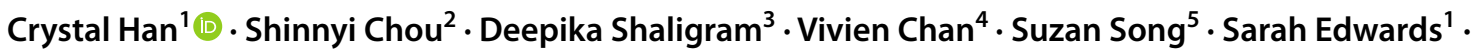 \\ Kimberly Gordon-Achebe ${ }^{1}$
}

Received: 26 July 2021 / Accepted: 8 November 2021 / Published online: 19 November 2021

This is a U.S. government work and not under copyright protection in the U.S.; foreign copyright protection may apply 2021

\section{To the Editor:}

The Asian American and Pacific Islander (AAPI) population is the fastest growing minority group in the United States [1]. The need for understanding AAPI experiences is particularly evident this past year given the increase in antiAAPI attacks amidst the COVID-19 pandemic. AAPI communities have high rates of depression and suicide attempts but are among the least likely to receive treatment, partly due to lack of culturally sensitive providers [2], perceived sense of burden to others, family shame, and stigma. Training psychiatrists in structural competency and cultural humility is more predictive of positive patient outcomes than ethnic matching [3], with such interventions including cultural awareness workshops and cultural curricula reported as part of various institutes' trainings [3, 4].

Literature regarding the implementation of cultural curricula appears limited, and none addresses issues critical to child and adolescent psychiatry (CAP) regarding AAPI, including child development and family systems. Despite $\mathrm{Lu}$ et al. discussing the importance of AAPI-focused mental health curricula 20 years ago [5], few psychiatry training programs include education on AAPI culture, needs and treatment, and fewer for child psychiatry trainees. We

Crystal Han

chan@som.umaryland.edu

1 University of Maryland School of Medicine, Baltimore, MD, USA

2 University of Pittsburgh Medical Center Western Psychiatric Hospital, Pittsburgh, PA, USA

3 Boston Children's Hospital, Boston, MA, USA

4 University of California, Irvine Student Health Center Irvine, California; and University of California Health Office of the President, sponsored by University of California San Diego Health, Irvine, CA, USA

5 George Washington University Medical Center, Washington, DC, USA describe a curriculum educating child psychiatry fellows on AAPI mental health, to increase cultural sensitivity and to delineate differences in psychoeducation approaches (de-jargoning to reduce stigma), explanatory models of illness, and family engagement.

Between October 2020 and April 2021, one psychiatry departmental virtual grand round and four child psychiatry fellow virtual lectures were given at the University of Maryland (Baltimore, MD) on culturally sensitive practices for AAPI youth and families. Learning objectives included 1) exploring cultural identity, 2) describing cultural humility, 3) identifying treatment needs, 4) reviewing challenges and guidance in treating AAPI youths and families, and 5) discussing race in supervision. The grand round lecture had 120 participants including psychiatry faculty, residents, subspecialty fellows, and other behavioral health providers. The virtual didactic lectures had 14 child psychiatry fellow attendees. Board certified psychiatrist guest lecturers with expertise in cultural psychiatry, four of whom specializing in AAPI issues spoke from locations including Boston, MA; San Francisco, CA; and Yakima, WA. This project was a quality improvement initiative and was not formally supervised by the Institutional Review Board per their policies.

The grand rounds lecture on cultural humility and antiracism explored cultural identity, health inequity, systemic racism, and differentiated concepts of cultural competence and cultural humility. Each child fellow lecture varied between 1 and 2 hours in a didactic format that included time for discussion. The first lecture explored cultural identities through the presenter's personal experiences, discussed diversity within AAPI including a timeline of immigration history, and discriminations against various ethnic groups. The second lecture explored treatment needs of AAPI youth, struggles within AAPI family systems, and intervention strategies including aligning with parental values (such as framing treatment as a tool toward academic success, and avoiding jargon such as diagnostic terms). The third lecture 
explored the model minority myth, anti-AAPI racism, AAPI family values and explanatory models of illness, cross-cultural and intergenerational challenges, AAPI mental health disparities and barriers to treatment, and outcome measures including the integration of a bicultural identity. The last lecture discussed a case study of an inter-racial supervisortrainee dyad's exploration of race during psychotherapy supervision, the importance of and obstacles to productive dialogs, and the resultant growth from this experience. While all the learners were CAP trainees, the curriculum is applicable to general psychiatry as well.

Eight child psychiatry fellows and one child psychiatry faculty provided feedback on survey questions including: "Based on what you've learned so far, what are some things that were most helpful or interesting?" "Did this lecture series deepen your understanding of the issues facing AAPI families?" and "Would you recommend we repeat this lecture series for future trainees?" There was a unanimous recommendation that the lectures be offered to future trainees, and all participants reported a deeper understanding of issues facing the AAPI community, youth and families. Responses reflected appreciation of knowledge obtained through the lectures including the challenges, struggles and stigma facing AAPI youths and families, cultural elements which enhanced the understanding of AAPI youths' mental health needs, and strategies to align with AAPI parents toward treatment goals. Participants shared that despite the importance of this subject, it was not discussed in their past training, and that it was helpful for both their current and future clinical practice. One participant reflected on the discomfort of discussing race in psychotherapy supervision, and the importance of disseminating these ideas to all supervisors.

The lack of a standardized curriculum in cultural psychiatry has resulted in a dearth of providers and mentors wellversed in culturally informed and sensitive care for AAPI communities. This is especially salient in CAP training, where culture is inextricably tied to AAPI youth development, and traditions influence the dynamics of AAPI family systems. For example, the model minority myth shapes AAPI identity development, inhibits help seeking and misleads policymakers to overlook struggles of AAPI families. Awareness of the way AAPI culture shapes illness beliefs and treatment preferences is paramount toward building rapport and engaging patients and families. Above, we describe a trial curriculum focused on the AAPI community, implemented within a single CAP fellowship training program. The overwhelming respondent recommendations for the curriculum to be repeated, has fueled the groundwork for further expansion of this curriculum.

Training psychiatrists in structural competency and cultural humility fosters greater understanding for sociocultural concepts and increases trainees' confidence in socioculturally-relevant skills and attitudes [4]. Additional lectures at this institution will include a case consultation lecture on AAPI patient cases, and a session on how to engage AAPI youths, families and communities in mental health literacy and treatment. Broader educational goals include the standardization and expansion of this curriculum to other institutions, in order to meet the critical unmet needs in general and child psychiatry training. This curriculum is just the beginning and we advocate for training programs to implement culturally sensitive clinical supervision and training experiences, and for researchers to increase AAPI inclusion in research samples so psychiatry educators and trainees can provide culturally sensitive treatment of AAPI youth.

Funding American Psychiatric Association (APA) Substance and Mental Health Services Administration (SAMHSA) Minority Fellowship Program.

\section{Declarations}

This project was undertaken as a quality improvement (QI) initiative and, as such, was not formally supervised by the Institutional Review Board per their policies.

Disclosure On behalf of all authors, the corresponding author states that there is no conflict of interest.

\section{References}

1. The Lancet. Racism in the USA: ensuring Asian American health equity. Lancet. 2021;397:1237.

2. Substance Abuse Mental Health Services Administration, Han B, Hedden S, Lipari R, Copello EAP, Kroutil LA. Center for Behavioral Health Statistics and Quality, Receipt of Services for Behavioral Health Problems: Results from the 2014 National Survey on Drug Use and Health. In: National Survey on drug use and health. 2015. https://www.samhsa.gov/data/sites/default/files/ NSDUH-DR-FRR3-2014/NSDUH-DR-FRR3-2014/NSDUH-DRFRR3-2014.htm. Accessed July 2021.

3. Kirmayer LJ, Jarvis GE. Culturally responsive services as a path to equity in mental healthcare. Healthc Pap. 2019;18(2):11-23.

4. Chen JA, Crawford C, Owusu M, Jahan AB, Faller V, Palmer C, Trinh N. Sociocultural psychiatry: developing and implementing a residency curriculum. Acad Psychiatry. 2021. https://doi.org/10. 1007/s40596-021-01491-2.

5. Lu FG, Du N, Gaw A, Lin KM. A psychiatric residency curriculum about Asian-American issues. Acad Psychiatry. 2002;26(4):225-36.

Publisher's Note Springer Nature remains neutral with regard to jurisdictional claims in published maps and institutional affiliations. 